\title{
STEADY-STATE CHARACTERISTICS OF THREE-CHANNEL QUEUEING SYSTEMS WITH ERLANGIAN SERVICE TIMES
}

\author{
Bohdan Kopytko ${ }^{1}$, Kostyantyn Zhernovyi ${ }^{2}$ \\ ${ }^{1}$ Institute of Mathematics, Czestochowa University of Technology \\ Częstochowa, Poland \\ ${ }^{2}$ Educational and Scientific Institute, Banking University \\ Lviv, Ukraine \\ bohdan.kopytko@im.pcz.pl,k.zhernovyi@yahoo.com
}

\begin{abstract}
We propose a method of study the $\mathrm{M} / \mathrm{E}_{2} / 3 / \infty$ queueing systems: standard system and systems with the threshold and hysteretic strategies of the random dropping of customers in order to control the input flow. Recurrence relations to compute the stationary distribution of the number of customers and the steady-state characteristics are obtained. The developed algorithms are tested on examples using simulation models constructed with the assistance of the GPSS World tools.
\end{abstract}

Keywords: three-channel queueing system, Poisson input, Erlangian service times, random dropping of customers, fictitious phase method, recurrence relations

\section{Introduction}

There are currently no created analytical methods of study of the $\mathrm{M} / \mathrm{G} / \mathrm{n} / \mathrm{m}$ and $\mathrm{M} / \mathrm{G} / \mathrm{n} / \infty$ queueing systems with the number of channels $n>1$. The $\mathrm{M} / \mathrm{D} / \mathrm{n} / \infty$ system is one of the few exceptions. It is possible to obtain some of the characteristics associated with the number of customers in the system [1].

To investigate the single-channel systems with Erlangian service times, in particular the $\mathrm{M} / \mathrm{E}_{\mathrm{s}} / 1 / \infty$ system [1], the method of fictitious phases, developed by Erlang [2], was applied. The Erlangian service times of the order $s$ means that each customer runs sequentially $s$ service phases, the duration of which is distributed exponentially with parameters $\mu_{1}, \mu_{2}, \ldots, \mu_{s}$ respectively.

The objective of this work is the construction with the help of the method of fictitious phase recurrence algorithms for computing the stationary distribution of the number of customers in the three-channel queueing system $\mathrm{M} / \mathrm{E}_{2} / 3 / \infty$, as well as in the systems of the same type with threshold and hysteretic strategies of the random dropping of customers. The random dropping of arrivals is a powerful tool for parameter control of a queueing system. Each arriving customer can be accepted for service with a probability depending on the queue length at the time of arrival of the customer, even if the buffer is not completely full [3-6]. 


\section{The $M / E_{2} / 3 / \infty$ system}

We consider the $\mathrm{M} / \mathrm{E}_{2} / 3 / \infty$ system. Let $\lambda$ be a parameter of the exponential distribution of the time intervals between moments of arrival of customers. Suppose that the service time of each customer is distributed under the generalized Erlang law of the second order, that is, the service time is the sum of two independent random variables exponentially distributed with parameters $\mu_{1}$ and $\mu_{2}$ respectively.

Let $n_{c}$ denote the number of customers in the system and let $n_{b c}$ be the number of busy channels. In accordance with the method of phases, let us enumerate the system's states as follows: $s_{0}$ corresponds to the empty system; $s_{1(10)}$ is the state, when $n_{c}=n_{b c}=1$ and the service occurs in the first phase; $s_{1(01)}$ is the state, when $n_{c}=n_{b c}=1$ and the service occurs in the second phase; $s_{2(20)}$ is the state, when $n_{c}=n_{b c}=2$ and the services occur in the first phase; $s_{2(11)}$ is the state, when $n_{c}=n_{b c}=2$ and the services occur in the first and second phase respectively; $s_{2(02)}$ is the state, when $n_{c}=n_{b c}=2$ and the services occur in the second phase; $s_{k(30)}$ is the state, when $n_{c}=k(k \geq 3), n_{b c}=3$ and the services occur in the first phase; $s_{k(21)}$ is the state, when $n_{c}=k(k \geq 3), n_{b c}=3$ and the services in two channels occur in the first phase and in one channel the service occurs in the second phase; $s_{k(12)}$ is the state, when $n_{c}=k(k \geq 3), n_{b c}=3$ and the service in two channels occur in the second phase and in one channel the service occurs in the first phase; $s_{k(03)}$ is the state, when $n_{c}=k(k \geq 3), n_{b c}=3$ and the service occurs in the second phase. We denote by $p_{0}, p_{1(10)}, p_{1(01)}, p_{2(20)}, p_{2(11)}, p_{2(02)}, p_{k(30)}, p_{k(21)}, p_{k(12)}$ and $p_{k(03)}(k \geq 3)$ respectively, steady-state probabilities that the system is in the each of these states. Assuming that $p_{2(30)}=p_{2(20)}, p_{2(21)}=p_{2(11)}, p_{2(12)}=p_{2(02)}$, to calculate the steady-state probabilities, we obtain the system of equations:

$$
\begin{aligned}
& -\lambda p_{0}+\mu_{2} p_{1(01)}=0, \\
& -\left(\lambda+\mu_{2}\right) p_{1(01)}+\mu_{1} p_{1(10)}+2 \mu_{2} p_{2(02)}=0, \\
& -\left(\lambda+2 \mu_{2}\right) p_{2(02)}+\mu_{1} p_{2(11)}+3 \mu_{2} p_{3(03)}=0, \\
& -\left(\lambda+3 \mu_{2}\right) p_{3(03)}+\mu_{1} p_{3(12)}=0, \\
& -\left(\lambda+\mu_{1}\right) p_{1(10)}+\lambda p_{0}+\mu_{2} p_{2(11)}=0, \\
& -\left(\lambda+2 \mu_{1}\right) p_{2(20)}+\lambda p_{1(10)}+\mu_{2} p_{3(21)}=0, \\
& -\left(\lambda+\mu_{1}+\mu_{2}\right) p_{2(11)}+\lambda p_{1(01)}+2 \mu_{2} p_{3(12)}+2 \mu_{1} p_{2(20)}=0
\end{aligned}
$$




$$
\begin{aligned}
&-\left(\lambda+3 \mu_{1}\right) p_{k(30)}+\lambda p_{k-1(30)}+\mu_{2} p_{k+1(21)}=0, \quad k \geq 3 \\
&-\left(\lambda+2 \mu_{1}+\mu_{2}\right) p_{k(21)}+\lambda p_{k-1(21)}+3 \mu_{1} p_{k(30)}+2 \mu_{2} p_{k+1(12)}=0, \quad k \geq 3 \\
&-\left(\lambda+\mu_{1}+2 \mu_{2}\right) p_{k(12)}+\lambda p_{k-1(12)}+2 \mu_{1} p_{k(21)}+3 \mu_{2} p_{k+1(03)}=0, \quad k \geq 3 \\
&-\left(\lambda+3 \mu_{2}\right) p_{k(03)}+\lambda p_{k-1(03)}+\mu_{1} p_{k(12)}=0, \quad k \geq 4 \\
& p_{0}+p_{1(10)}+p_{1(01)}+p_{2(20)}+p_{2(11)}+p_{2(02)}+\sum_{k=3}^{\infty}\left(p_{k(30)}+p_{k(21)}+p_{k(12)}+p_{k(03)}\right)=1
\end{aligned}
$$

The steady-state distribution of the number of customers in the $M / E_{2} / 3 / \infty$ system exists under the condition that $\lambda \mathbf{E}\left(T_{\mathrm{sv}}\right)<3$, where $\mathbf{E}\left(T_{\mathrm{sv}}\right)=\frac{\mu_{1}+\mu_{2}}{\mu_{1} \mu_{2}}$ is the average service time per customer.

Introducing the notation

$$
\begin{aligned}
& \alpha_{i}=\frac{\lambda}{\mu_{i}}, i=1,2 ; \eta=\frac{\mu_{2}}{\mu_{1}} ; \tilde{p}_{1(10)}=\frac{p_{1(10)}}{p_{0}}, \tilde{p}_{1(01)}=\frac{p_{1(01)}}{p_{0}}, \tilde{p}_{2(20)}=\frac{p_{2(20)}}{p_{0}}=p, \tilde{p}_{2(11)}=\frac{p_{2(11)}}{p_{0}}, \\
& \tilde{p}_{2(02)}=\frac{p_{2(02)}}{p_{0}} ; \tilde{p}_{k(30)}=\frac{p_{k(30)}}{p_{0}}, \tilde{p}_{k(21)}=\frac{p_{k(21)}}{p_{0}}, \tilde{p}_{k(12)}=\frac{p_{k(12)}}{p_{0}}, \tilde{p}_{k(03)}=\frac{p_{k(03)}}{p_{0}}, k \geq 3,
\end{aligned}
$$

using equations (1) we find:

$$
\begin{aligned}
& \tilde{p}_{1(01)}=\alpha_{2}, \quad \tilde{p}_{3(03)}=\frac{A\left(\eta, \alpha_{1}, \alpha_{2}, p\right)}{B\left(\eta, \alpha_{1}, \alpha_{2}\right)}, \quad \tilde{p}_{3(12)}=\left(\alpha_{1}+3 \eta\right) \tilde{p}_{3(03)}, \\
& \tilde{p}_{2(11)}=\frac{2 \eta\left(\alpha_{1}+3 \eta\right) \tilde{p}_{3(03)}+2 p+\eta \alpha_{2}^{2}}{\eta\left(\alpha_{2}+1\right)+1}, \\
& \tilde{p}_{2(02)}=\frac{\eta\left(2 \alpha_{1}+3 \eta \alpha_{2}+9 \eta+3\right) \tilde{p}_{3(03)}+2 p+\eta \alpha_{2}^{2}}{\left(\alpha_{1}+2 \eta\right)\left(\eta\left(\alpha_{2}+1\right)+1\right)}, \\
& \tilde{p}_{1(10)}=\alpha_{2}\left(\alpha_{1}+\eta\right)-2 \eta \tilde{p}_{2(02)}, \quad \tilde{p}_{3(21)}=\frac{1}{\eta}\left(\left(\alpha_{1}+2\right) p-\alpha_{1} \tilde{p}_{1(10)}\right),
\end{aligned}
$$

where

$$
\begin{gathered}
A\left(\eta, \alpha_{1}, \alpha_{2}, p\right)=\alpha_{2}\left(\alpha_{1}+1\right)\left(\left(\alpha_{1}+\eta\right)\left(\alpha_{1}+2 \eta\right)\left(\eta \alpha_{2}+\eta+1\right)-2 \eta^{2} \alpha_{2}\right)- \\
-\alpha_{1}\left(\alpha_{1}+2 \eta\right)\left(\eta \alpha_{2}+\eta+1\right)-\eta^{2} \alpha_{2}^{2}\left(\alpha_{1}+2 \eta\right)-2 \eta\left(3 \alpha_{1}+2 \eta+2\right) p \\
B\left(\eta, \alpha_{1}, \alpha_{2}\right)=2 \eta^{2}\left(\left(\alpha_{1}+1\right)\left(2 \alpha_{1}+3 \eta \alpha_{2}+9 \eta+3\right)+\left(\alpha_{1}+2 \eta\right)\left(\alpha_{1}+3 \eta\right)\right) .
\end{gathered}
$$

Recurrence relations (4) contain the parameter $p=\tilde{p}_{2(20)}$ to be determined. 
From equations (2) we obtain the recurrence relations:

$$
\begin{aligned}
& \tilde{p}_{k(03)}=f_{03}\left(\tilde{p}_{k-1(12)}, \tilde{p}_{k-2(12)}, \tilde{p}_{k-1(21)}\right), \quad k \geq 4 ; \\
& \tilde{p}_{k(12)}=f_{12}\left(\tilde{p}_{k(03)}, \tilde{p}_{k-1(03)}\right), \quad k \geq 4 ; \\
& \tilde{p}_{k(30)}=f_{30}\left(\tilde{p}_{k(21)}, \tilde{p}_{k-1(21)}, \tilde{p}_{k+1(12)}\right), \quad k \geq 3 ; \\
& \tilde{p}_{k(21)}=f_{21}\left(\tilde{p}_{k-1(30)}, \tilde{p}_{k-2(30)}\right), \quad k \geq 4,
\end{aligned}
$$

where

$$
\begin{array}{ll}
f_{03}(x, y, z)=\frac{1}{3 \eta}\left(\left(\alpha_{1}+2 \eta+1\right) x-\alpha_{1} y-2 z\right), & f_{12}(x, y)=\left(\alpha_{1}+3 \eta\right) x-\alpha_{1} y, \\
f_{30}(x, y, z)=\frac{1}{3}\left(\left(\alpha_{1}+\eta+2\right) x-\alpha_{1} y-2 \eta z\right), & f_{21}(x, y)=\frac{1}{\eta}\left(\left(\alpha_{1}+3\right) x-\alpha_{1} y\right) .
\end{array}
$$

The system (1)-(3) consists of an infinite number of equations. Let $N$ be a sufficiently large natural number. Writing the normalization condition (3) in the form

$$
\begin{gathered}
p_{0(N)}+p_{1(10)(N)}+p_{1(01)(N)}+p_{2(20)(N)}+p_{2(11)(N)}+p_{2(02)(N)}+ \\
+\sum_{k=3}^{N}\left(p_{k(30)(N)}+p_{k(21)(N)}+p_{k(12)(N)}+p_{k(03)(N)}\right)=1,
\end{gathered}
$$

we determine the approximate values of the stationary probabilities using equations

$$
\begin{aligned}
& \frac{1}{p_{0(N)}}=1+\tilde{p}_{1(10)(N)}+\tilde{p}_{1(01)(N)}+\tilde{p}_{2(20)(N)}+\tilde{p}_{2(11)(N)}+\tilde{p}_{2(02)(N)}+ \\
& \quad+\sum_{k=3}^{N}\left(\tilde{p}_{k(30)(N)}+\tilde{p}_{k(21)(N)}+\tilde{p}_{k(12)(N)}+\tilde{p}_{k(03)(N)}\right) ; \quad p_{1(10)(N)}=p_{0(N)} \tilde{p}_{1(10)(N)}, \\
& p_{1(01)(N)}=p_{0(N)} \tilde{p}_{1(01)(N)}, \quad p_{2(20)(N)}=p_{0(N)} \tilde{p}_{2(20)(N)}, \quad p_{2(11)(N)}=p_{0(N)} \tilde{p}_{2(11)(N)}, \\
& p_{2(02)(N)}=p_{0(N)} \tilde{p}_{2(02)(N)} ; \quad p_{k(30)(N)}=p_{0(N)} \tilde{p}_{k(30)(N)}, \quad p_{k(21)(N)}=p_{0(N)} \tilde{p}_{k(21)(N)}, \\
& p_{k(12)(N)}=p_{0(N)} \tilde{p}_{k(12)(N)}, \quad p_{k(03)(N)}=p_{0(N)} \tilde{p}_{k(03)(N)}, \quad 3 \leq k \leq N ; \\
& p_{1(N)}=p_{1(10)(N)}+p_{1(01)(N)}, \quad p_{2(N)}=p_{2(20)(N)}+p_{2(11)(N)}+p_{2(02)(N)} ; \\
& p_{k(N)}=p_{k(30)(N)}+p_{k(21)(N)}+p_{k(12)(N)}+p_{k(03)(N)}, \quad 3 \leq k \leq N .
\end{aligned}
$$

Here $p_{k}$ is the steady-state probability that $n_{c}=k, p_{k(N)}$ is the approximate value of the probability $p_{k}$, obtained after replacing condition (3) by equality (6).

Recurrence relations (4) and (5) allow us to calculate $\tilde{p}_{1(01)}$ and consistently obtain the expression for $\tilde{p}_{1(10)}, \tilde{p}_{2(11)}, \tilde{p}_{2(02)}, \tilde{p}_{k(30)}, \tilde{p}_{k(21)}, \tilde{p}_{k(12)}$ and $\tilde{p}_{k(03)}$ $(3 \leq k \leq N)$ as functions of the unknown parameter $p=\tilde{p}_{2(20)}$. To determine $p$, we 
use the condition that in the steady state, the average number of busy channels is equal to the system load factor $\lambda \mathbf{E}\left(T_{\mathrm{sv}}\right)=p_{1}+2 p_{2}+3 \sum_{k=3}^{\infty} p_{k}$, writing it in the form

$$
\lambda \mathbf{E}\left(T_{\mathrm{sv}}\right)=p_{1(N)}+2 p_{2(N)}+3 \sum_{k=3}^{N} p_{k(N)} .
$$

Using the formulas

$$
\mathbf{E}(Q)_{(N)}=p_{4(N)}+\sum_{k=5}^{N}(k-3) p_{k(N)}, \quad \mathbf{E}(W)_{(N)}=\frac{\mathbf{E}(Q)_{(N)}}{\lambda},
$$

we find approximate values of the average queue length $\mathbf{E}(Q)$ and the average waiting time $\mathbf{E}(W)$ in the steady state. The number $N$ is chosen so large that the condition

$$
\mathbf{E}(Q)_{(N)}-\mathbf{E}(Q)_{(N-1)}<\varepsilon
$$

holds, where $\varepsilon$ is a positive number specifying the required accuracy of calculations.

\section{The $M / E_{2} / 3 / \infty$ system with the threshold strategy of the random dropping of customers}

We consider for the $\mathrm{M} / \mathrm{E}_{2} / 3 / \infty$ system a strategy of random dropping of customers performed according to the rule: if at the time of the arrival of a customer $n_{c}=n$ (not taking into account the arrived customer), then the customer is accepted for service with probability $\beta_{n}\left(0<\beta_{n} \leq 1\right)$ and leaves the system (is discarded) with probability $1-\beta_{n}$. Confining ourselves to a simplified version of the strategy, let's fix a threshold value $h(h \geq 5)$ and suppose that $\beta_{n}=1$ for $1 \leq n \leq h-1$, and $\beta_{n}=\beta(0<\beta<1)$ for $n \geq h$. The intensity of the simplest flow of customers received for service as a result of random dropping, is equal to $\tilde{\lambda}=\lambda \beta$.

The system of equations for determining the steady-state probabilities contains the equation (1), (3) and the following equations:

$$
\begin{aligned}
& -\left(\lambda+3 \mu_{1}\right) p_{k(30)}+\lambda p_{k-1(30)}+\mu_{2} p_{k+1(21)}=0, \quad 3 \leq k \leq h-1 ; \\
& -\left(\lambda+2 \mu_{1}+\mu_{2}\right) p_{k(21)}+\lambda p_{k-1(21)}+3 \mu_{1} p_{k(30)}+2 \mu_{2} p_{k+1(12)}=0, \quad 3 \leq k \leq h-1 \text {; } \\
& -\left(\lambda+\mu_{1}+2 \mu_{2}\right) p_{k(12)}+\lambda p_{k-1(12)}+2 \mu_{1} p_{k(21)}+3 \mu_{2} p_{k+1(03)}=0, \quad 3 \leq k \leq h-1 ; \\
& -\left(\lambda+3 \mu_{2}\right) p_{k(03)}+\lambda p_{k-1(03)}+\mu_{1} p_{k(12)}=0, \quad 4 \leq k \leq h-1 \text {; }
\end{aligned}
$$




$$
\begin{aligned}
& -\left(\tilde{\lambda}+3 \mu_{1}\right) p_{h(30)}+\lambda p_{h-1(30)}+\mu_{2} p_{h+1(21)}=0, \\
& -\left(\tilde{\lambda}+2 \mu_{1}+\mu_{2}\right) p_{h(21)}+\lambda p_{h-1(21)}+3 \mu_{1} p_{h(30)}+2 \mu_{2} p_{h+1(12)}=0, \\
& -\left(\tilde{\lambda}+\mu_{1}+2 \mu_{2}\right) p_{h(12)}+\lambda p_{h-1(12)}+2 \mu_{1} p_{h(21)}+3 \mu_{2} p_{h+1(03)}=0, \\
& -\left(\tilde{\lambda}+3 \mu_{2}\right) p_{h(03)}+\lambda p_{h-1(03)}+\mu_{1} p_{h(12)}=0 ; \\
& -\left(\tilde{\lambda}+3 \mu_{1}\right) p_{k(30)}+\tilde{\lambda} p_{k-1(30)}+\mu_{2} p_{k+1(21)}=0, \quad h+1 \leq k \leq m+2 ; \\
& -\left(\tilde{\lambda}+2 \mu_{1}+\mu_{2}\right) p_{k(21)}+\tilde{\lambda} p_{k-1(21)}+3 \mu_{1} p_{k(30)}+2 \mu_{2} p_{k+1(12)}=0, \quad k \geq h+1 ; \\
& -\left(\tilde{\lambda}+\mu_{1}+2 \mu_{2}\right) p_{k(12)}+\tilde{\lambda} p_{k-1(12)}+2 \mu_{1} p_{k(21)}+3 \mu_{2} p_{k+1(03)}=0, \quad k \geq h+1 ; \\
& -\left(\tilde{\lambda}+3 \mu_{2}\right) p_{k(03)}+\tilde{\lambda} p_{k-1(03)}+\mu_{1} p_{k(12)}=0, \quad k \geq h+1 .
\end{aligned}
$$

Using equations (1), we have the equalities (4) and from the equations (9) we obtain the recurrence relations:

$$
\begin{aligned}
& \tilde{p}_{k(03)}=f_{03}\left(\tilde{p}_{k-1(12)}, \tilde{p}_{k-2(12)}, \tilde{p}_{k-1(21)}\right), \quad 4 \leq k \leq h ; \\
& \tilde{p}_{k(12)}=f_{12}\left(\tilde{p}_{k(03)}, \tilde{p}_{k-1(03)}\right), \quad 4 \leq k \leq h-1 ; \\
& \tilde{p}_{k(30)}=f_{30}\left(\tilde{p}_{k(21)}, \tilde{p}_{k-1(21)}, \tilde{p}_{k+1(12)}\right), \quad 3 \leq k \leq h-1 ; \\
& \tilde{p}_{k(21)}=f_{21}\left(\tilde{p}_{k-1(30)}, \tilde{p}_{k-2(30)}\right), \quad 4 \leq k \leq h,
\end{aligned}
$$

Using equation (10), we find:

$$
\begin{aligned}
& \tilde{p}_{h(12)}=\left(\tilde{\alpha}_{1}+3 \eta\right) \tilde{p}_{h(03)}-\alpha_{1} \tilde{p}_{h-1(03)}, \\
& \tilde{p}_{h+1(03)}=\frac{1}{3 \eta}\left(\left(\tilde{\alpha}_{1}+2 \eta+1\right) \tilde{p}_{h(12)}-\alpha_{1} \tilde{p}_{h-1(12)}-2 \tilde{p}_{h(21)}\right), \\
& \tilde{p}_{h(30)}=\frac{1}{3}\left(\left(\tilde{\alpha}_{1}+\eta+2\right) \tilde{p}_{h(21)}-\alpha_{1} \tilde{p}_{h-1(21)}-2 \eta \tilde{p}_{h+1(12)}\right), \\
& \tilde{p}_{h+1(21)}=\frac{1}{\eta}\left(\left(\tilde{\alpha}_{1}+3\right) \tilde{p}_{h(30)}-\alpha_{1} \tilde{p}_{h-1(30)}\right),
\end{aligned}
$$

where $\tilde{\alpha}_{i}=\frac{\tilde{\lambda}}{\mu_{i}}, i=1,2$. From equations (11) we obtain:

$$
\begin{aligned}
& \tilde{p}_{k(03)}=g_{03}\left(\tilde{p}_{k-1(12)}, \tilde{p}_{k-2(12)}, \tilde{p}_{k-1(21)}\right), \quad k \geq h+2 ; \\
& \tilde{p}_{k(12)}=g_{12}\left(\tilde{p}_{k(03)}, \tilde{p}_{k-1(03)}\right), \quad k \geq h+1 ; \\
& \tilde{p}_{k(30)}=g_{30}\left(\tilde{p}_{k(21)}, \tilde{p}_{k-1(21)}, \tilde{p}_{k+1(12)}\right), \quad k \geq h+1 ; \\
& \tilde{p}_{k(21)}=g_{21}\left(\tilde{p}_{k-1(30)}, \tilde{p}_{k-2(30)}\right), \quad k \geq h+2,
\end{aligned}
$$


where

$$
\begin{array}{ll}
g_{03}(x, y, z)=\frac{1}{3 \eta}\left(\left(\tilde{\alpha}_{1}+2 \eta+1\right) x-\tilde{\alpha}_{1} y-2 z\right), & g_{12}(x, y)=\left(\tilde{\alpha}_{1}+3 \eta\right) x-\tilde{\alpha}_{1} y, \\
g_{30}(x, y, z)=\frac{1}{3}\left(\left(\tilde{\alpha}_{1}+\eta+2\right) x-\tilde{\alpha}_{1} y-2 \eta z\right), & g_{21}(x, y)=\frac{1}{\eta}\left(\left(\tilde{\alpha}_{1}+3\right) x-\tilde{\alpha}_{1} y\right) .
\end{array}
$$

Recurrence relations (4), (12)-(14) allow us to calculate $\tilde{p}_{1(01)}$ and consistently obtain the expression for $\tilde{p}_{1(10)}, \tilde{p}_{2(11)}, \tilde{p}_{2(02)}, \tilde{p}_{k(30)}, \tilde{p}_{k(21)}, \tilde{p}_{k(12)}$ and $\tilde{p}_{k(03)}(k \geq 3)$ as functions of the unknown parameter $p=\tilde{p}_{2(20)}$. To determine $p$, we use the condition that in the steady state the average number of busy channels is equal to the system load factor $\mathbf{E}\left(T_{\mathrm{sv}}\right)\left(\lambda \sum_{k=0}^{h-1} p_{k}+\tilde{\lambda}\left(1-\sum_{k=0}^{h-1} p_{k}\right)\right)=p_{1}+2 p_{2}+3 \sum_{k=3}^{\infty} p_{k}$.

The steady-state probabilities exist under the condition that $\tilde{\lambda} \mathbf{E}\left(T_{\mathrm{sv}}\right)<3$. We find the approximate values of steady-state probabilities by the formulas (7) where the number $N$ is chosen so large that the condition (8) holds.

The approximate values of the average queue length $\mathbf{E}(Q)$, average waiting time $\mathbf{E}(W)$ and probability of service $\mathbf{P}_{\mathrm{sv}}$ in the steady state

Using the formulas

$$
\begin{gathered}
\mathbf{E}(Q)_{(N)}=p_{4(N)}+\sum_{k=5}^{N}(k-3) p_{k(N)}, \quad \mathbf{E}(W)_{(N)}=\frac{\mathbf{E}(Q)_{(N)}}{\lambda \mathbf{P}_{\mathrm{sv}(N)}}, \\
\mathbf{P}_{\mathrm{sv}(N)}=\frac{3\left(1-p_{0(N)}\right)-2 p_{1(N)}-p_{2(N)}}{\alpha_{2}(\eta+1)},
\end{gathered}
$$

we find the approximate values of the average queue length $\mathbf{E}(Q)$, average waiting time $\mathbf{E}(W)$ and probability of service $\mathbf{P}_{\mathrm{sv}}$ in the steady state. The formula for $\mathbf{P}_{\mathrm{sv}}$ is obtained as the ratio of the average number of serviced customers per unit of time to the average arrival rate of customers.

\section{The $M / E_{2} / 3 / \infty$ system with the hysteretic strategy of the random dropping of customers}

We consider for the $\mathrm{M} / \mathrm{E}_{2} / 3 / \infty$ system a hysteretic strategy of a random dropping of customers with two thresholds $h_{1}$ and $h_{2}\left(h_{2}>h_{1} \geq 4, h_{2}-h_{1} \geq 3\right)$ and with two operation modes: basic mode and dropping mode. Assume that $\beta_{n}=1,1 \leq n \leq h_{1}$, for the basic mode, and $\beta_{n}=\beta(0<\beta<1), n \geq h_{2}$, for the dropping mode. Here $n$ is the number of customers in the system at the time of the arrival of a customer 
(not taking into account the arrived customer). If at the time of the arrival of a customer, condition $h_{1}<n<h_{2}$ is satisfied, then the mode is not changed. The dropping mode operates from the time when the number of customers in the system (on service and in the queue) reaches the value of $h_{2}$, to the time when the number of customers decreases to $h_{1}$.

We introduce the following notation for the states of the system in the basic mode: the states $s_{0}, s_{1(10)}, s_{1(01)}, s_{2(20)}, s_{2(11)}, s_{2(02)}$ correspond to the notation introduced above, $s_{k(30)}$ is the state, when $n_{c}=k\left(3 \leq k \leq h_{2}-1\right), n_{b c}=3$ and the services occur in the first phase; $s_{k(21)}$ is the state, when $n_{c}=k\left(3 \leq k \leq h_{2}-1\right), n_{b c}=3$ and the services in two channels occur in the first phase and in one channel the service occurs in the second phase; $s_{k(12)}$ is the state, when $n_{c}=k\left(3 \leq k \leq h_{2}-1\right), n_{b c}=3$ and the service in two channels occur in the second phase and in one channel the service occurs in the first phase; $s_{k(03)}$ is the state, when $n_{c}=k\left(3 \leq k \leq h_{2}-1\right), n_{b c}=3$ and the service occurs in the second phase. We denote by $p_{0}, p_{1(10)}, p_{1(01)}, p_{2(20)}, p_{2(11)}$, $p_{2(02)}, p_{k(30)}, p_{k(21)}, p_{k(12)}$ and $p_{k(03)}\left(3 \leq k \leq h_{2}-1\right)$ respectively, the steady-state probabilities that the system is in each of mentioned states. We denote by $\tilde{s}_{k(30)}, \tilde{s}_{k(21)}, \tilde{s}_{k(12)}$ and $\tilde{s}_{k(03)}\left(k \geq h_{1}+1\right)$ analogous states of the system in the dropping mode and let $q_{k(30)}, q_{k(21)}, q_{k(12)}$ and $q_{k(03)}\left(k \geq h_{1}+1\right)$ denote the steady-state probabilities that the system is in each of these states.

For determining the steady-state probabilities, we obtain the system containing the equation (1) and the following equations:

$$
\begin{gathered}
-\left(\lambda+3 \mu_{1}\right) p_{k(30)}+\lambda p_{k-1(30)}+\mu_{2} p_{k+1(21)}=0 \\
3 \leq k \leq h_{1}-1, h_{1}+1 \leq k \leq h_{2}-2 ; \\
-\left(\lambda+2 \mu_{1}+\mu_{2}\right) p_{k(21)}+\lambda p_{k-1(21)}+3 \mu_{1} p_{k(30)}+2 \mu_{2} p_{k+1(12)}=0 \\
3 \leq k \leq h_{1}-1, h_{1}+1 \leq k \leq h_{2}-2 ; \\
-\left(\lambda+\mu_{1}+2 \mu_{2}\right) p_{k(12)}+\lambda p_{k-1(12)}+2 \mu_{1} p_{k(21)}+3 \mu_{2} p_{k+1(03)}=0, \\
3 \leq k \leq h_{1}-1, h_{1}+1 \leq k \leq h_{2}-2 ; \\
-\left(\lambda+3 \mu_{2}\right) p_{k(03)}+\lambda p_{k-1(03)}+\mu_{1} p_{k(12)}=0, \quad 4 \leq k \leq h_{2}-1 ; \\
-\left(\lambda+3 \mu_{1}\right) p_{h_{1}(30)}+\lambda p_{h_{1}-1(30)}+\mu_{2}\left(p_{h_{1}+1(21)}+q_{h_{1}+1(21)}\right)=0, \\
-\left(\lambda+2 \mu_{1}+\mu_{2}\right) p_{h_{1}(21)}+\lambda p_{h_{1}-1(21)}+3 \mu_{1} p_{h_{1}(30)}+2 \mu_{2}\left(p_{h_{1}+1(12)}+q_{h_{1}+1(12)}\right)=0, \\
-\left(\lambda+\mu_{1}+2 \mu_{2}\right) p_{h_{1}(12)}+\lambda p_{h_{1}-1(12)}+2 \mu_{1} p_{h_{1}(21)}+3 \mu_{2}\left(p_{h_{1}+1(03)}+q_{h_{1}+1(03)}\right)=0 ;
\end{gathered}
$$




$$
\begin{gathered}
-\left(\lambda+3 \mu_{1}\right) p_{h_{2}-1(30)}+\lambda p_{h_{2}-2(30)}=0 \\
-\left(\lambda+2 \mu_{1}+\mu_{2}\right) p_{h_{2}-1(21)}+\lambda p_{h_{2}-2(21)}+3 \mu_{1} p_{h_{2}-1(30)}=0 \\
-\left(\lambda+\mu_{1}+2 \mu_{2}\right) p_{h_{2}-1(12)}+\lambda p_{h_{2}-2(12)}+2 \mu_{1} p_{h_{2}-1(21)}=0 \\
-\left(\tilde{\lambda}+3 \mu_{1}\right) q_{h_{1}+1(30)}+\mu_{2} q_{h_{1}+2(21)}=0 \\
-\left(\tilde{\lambda}+2 \mu_{1}+\mu_{2}\right) q_{h_{1}+1(21)}+3 \mu_{1} q_{h_{1}+1(30)}+2 \mu_{2} q_{h_{1}+2(12)}=0 \\
-\left(\tilde{\lambda}+\mu_{1}+2 \mu_{2}\right) q_{h_{1}+1(12)}+2 \mu_{1} q_{h_{1}+1(21)}+3 \mu_{2} q_{h_{1}+2(03)}=0 \\
-\left(\tilde{\lambda}+3 \mu_{2}\right) q_{h_{1}+1(03)}+\mu_{1} q_{h_{1}+1(12)}=0 \\
-\left(\tilde{\lambda}+2 \mu_{1}+\mu_{2}\right) q_{k(21)}+\tilde{\lambda} q_{k-1(21)}+3 \mu_{1} q_{k(30)}+2 \mu_{2} q_{k+1(12)}=0, h_{1}+2 \leq k \leq h_{2}-1, k \geq h_{2}+1 ; \\
-\left(\tilde{\lambda}+\mu_{1}+2 \mu_{2}\right) q_{k(12)}+\tilde{\lambda} q_{k-1(12)}+2 \mu_{1} q_{k(21)}+3 \mu_{2} q_{k+1(03)}=0, h_{1}+2 \leq k \leq h_{2}-1, k \geq h_{2}+1 ; \\
-\left(\tilde{\lambda}+3 \mu_{2}\right) q_{k(03)}+\tilde{\lambda} q_{k-1(03)}+\mu_{1} q_{k(12)}=0, h_{1}+2 \leq k \leq h_{2}-1, k \geq h_{2}+1 ; \\
+\sum_{k=h_{1}+1}^{\infty}\left(q_{k(30)}+q_{k(21)}+q_{k(12)}+q_{k(03)}\right)=1 \\
-\left(\tilde{\lambda}+3 \mu_{1}\right) q_{h_{2}(30)}+\tilde{\lambda} q_{h_{2}-1(30)}+\lambda p_{h_{2}-1(30)}+\mu_{2} q_{h_{2}+1(21)}=0 \\
-\left(\tilde{\lambda}+2 \mu_{1}+\mu_{2}\right) q_{h_{2}(21)}+\tilde{\lambda} q_{h_{2}-1(21)}+\lambda p_{h_{2}-1(21)}+3 \mu_{1} q_{h_{2}(30)}+2 \mu_{2} q_{h_{2}+1(12)}=0 \\
-\left(\tilde{\lambda}+\mu_{1}+2 \mu_{2}\right) q_{h_{2}(12)}+\tilde{\lambda} q_{h_{2}-1(12)}+\lambda p_{h_{2}-1(12)}+2 \mu_{1} q_{h_{2}(21)}+3 \mu_{2} q_{h_{2}+1(03)}=0 \\
-\left(\tilde{\lambda}+3 \mu_{2}\right) q_{h_{2}(03)}+\tilde{\lambda} q_{h_{2}-1(03)}+\lambda p_{h_{2}-1(03)}+\mu_{1} q_{h_{2}(12)}=0 \\
p_{0}+p_{1(10)}+p_{1(01)}+p_{2(20)}+p_{2(11)}+p_{2(02)}+\sum_{k=3}\left(p_{k(30)}+p_{k(21)}+p_{k(12)}+p_{k(03)}\right)+
\end{gathered}
$$

Introduce the notation

$$
\begin{aligned}
& \tilde{q}_{k(30)}=\frac{q_{k(30)}}{p_{0}}, \quad \tilde{q}_{k(21)}=\frac{q_{k(21)}}{p_{0}}, \quad \tilde{q}_{k(12)}=\frac{q_{k(12)}}{p_{0}}, \quad \tilde{q}_{k(03)}=\frac{q_{k(03)}}{p_{0}}, k \geq h_{1}+1 ; \\
& \tilde{p}_{2(20)}=p, \quad \tilde{q}_{h_{1}+1(03)}=q, \quad \tilde{q}_{h_{1}+1(21)}=r .
\end{aligned}
$$

Using equations (1), we have the equalities (4) and from the equations (16) we obtain the recurrence relations: 


$$
\begin{aligned}
& \tilde{p}_{k(03)}=f_{03}\left(\tilde{p}_{k-1(12)}, \tilde{p}_{k-2(12)}, \tilde{p}_{k-1(21)}\right), \quad 4 \leq k \leq h_{1}, h_{1}+2 \leq k \leq h_{2}-1 ; \\
& \tilde{p}_{k(12)}=f_{12}\left(\tilde{p}_{k(03)}, \tilde{p}_{k-1(03)}\right), \quad 4 \leq k \leq h_{2}-1 ; \\
& \tilde{p}_{k(30)}=f_{30}\left(\tilde{p}_{k(21)}, \tilde{p}_{k-1(21)}, \tilde{p}_{k+1(12)}\right), \quad 3 \leq k \leq h_{1}-1, h_{1}+1 \leq k \leq h_{2}-2 ; \\
& \tilde{p}_{k(21)}=f_{21}\left(\tilde{p}_{k-1(30)}, \tilde{p}_{k-2(30)}\right), \quad 4 \leq k \leq h_{1}, h_{1}+2 \leq k \leq h_{2}-1 ;
\end{aligned}
$$

Using equations (17), (21), (22) and (18), we find:

$$
\begin{aligned}
& \tilde{p}_{h_{1}+1(03)}=\frac{1}{3 \eta}\left(\left(\alpha_{1}+2 \eta+1\right) \tilde{p}_{h_{1}(12)}-\alpha_{1} \tilde{p}_{h_{1}-1(12)}-2 \tilde{p}_{h_{1}(21)}-3 \eta q\right) \\
& \tilde{q}_{h_{1}+1(12)}=\left(\tilde{\alpha}_{1}+3 \eta\right) q, \quad \tilde{q}_{h_{1}+2(03)}=\frac{1}{3 \eta}\left(\left(\tilde{\alpha}_{1}+2 \eta+1\right) \tilde{q}_{h_{1}+1(12)}-2 r\right) \\
& \tilde{q}_{h_{1}+1(30)}=\frac{1}{3}\left(\left(\tilde{\alpha}_{1}+\eta+2\right) r-2 \eta \tilde{q}_{h_{1}+2(12)}\right), \quad \tilde{q}_{h_{1}+2(21)}=\frac{\tilde{\alpha}_{1}+3}{\eta} \tilde{q}_{h_{1}+1(30)} ; \\
& \tilde{q}_{k(03)}=g_{03}\left(\tilde{q}_{k-1(12)}, \tilde{q}_{k-2(12)}, \tilde{q}_{k-1(21)}\right), \quad h_{1}+3 \leq k \leq h_{2}, \quad k \geq h_{2}+2 ; \\
& \tilde{q}_{k(12)}=g_{12}\left(\tilde{q}_{k(03)}, \tilde{q}_{k-1(03)}\right), \quad h_{1}+2 \leq k \leq h_{2}-1, \quad k \geq h_{2}+1 ; \\
& \tilde{q}_{k(30)}=g_{30}\left(\tilde{q}_{k(21)}, \tilde{q}_{k-1(21)}, \tilde{q}_{k+1(12)}\right), \quad h_{1}+2 \leq k \leq h_{2}-1, \quad k \geq h_{2}+1 ; \\
& \tilde{q}_{k(21)}=g_{21}\left(\tilde{q}_{k-1(30)}, \tilde{q}_{k-2(30)}\right), \quad h_{1}+3 \leq k \leq h_{2}, \quad k \geq h_{2}+2 ; \\
& \tilde{p}_{h_{1}(30)}=\frac{1}{3}\left(\left(\alpha_{1}+\eta+2\right) \tilde{p}_{h_{1}(21)}-\alpha_{1} \tilde{p}_{h_{1}-1(21)}-2 \eta\left(\tilde{p}_{h_{1}+1(12)}+\tilde{q}_{h_{1}+1(12)}\right)\right. \\
& \tilde{p}_{h_{1}+1(21)}=\frac{1}{\eta}\left(\left(\alpha_{1}+3\right) \tilde{p}_{h_{1}(30)}-\alpha_{1} \tilde{p}_{h_{1}-1(30)}-\eta r\right), \quad \tilde{p}_{h_{2}-1(30)}=\frac{\alpha_{1}}{\alpha_{1}+3} \tilde{p}_{h_{2}-2(30)} .
\end{aligned}
$$

To determine $q$ and $r$ as functions of the parameter $p$ we use the equations (19) and (20).

The equations (23) allow us to find

$$
\begin{aligned}
& \tilde{q}_{h_{2}(12)}=\left(\tilde{\alpha}_{1}+3 \eta\right) \tilde{q}_{h_{2}(03)}-\tilde{\alpha}_{1} \tilde{q}_{h_{2}-1(03)}-\alpha_{1} \tilde{p}_{h_{2}-1(03)}, \\
& \tilde{q}_{h_{2}+1(03)}=\left(\left(\tilde{\alpha}_{1}+2 \eta+1\right) \tilde{q}_{h_{2}(12)}-\tilde{\alpha}_{1} \tilde{q}_{h_{2}-1(12)}-\alpha_{1} \tilde{p}_{h_{2}-1(12)}-2 \tilde{q}_{h_{2}(21)}\right) /(3 \eta), \\
& \tilde{q}_{h_{2}(30)}=\frac{1}{3}\left(\left(\tilde{\alpha}_{1}+\eta+2\right) \tilde{q}_{h_{2}(21)}-\tilde{\alpha}_{1} \tilde{q}_{h_{2}-1(21)}-\alpha_{1} \tilde{p}_{h_{2}-1(21)}-2 \eta \tilde{q}_{h_{2}+1(12)}\right), \\
& \tilde{q}_{h_{2}+1(21)}=\frac{1}{\eta}\left(\left(\tilde{\alpha}_{1}+3\right) \tilde{q}_{h_{2}(30)}-\tilde{\alpha}_{1} \tilde{q}_{h_{2}-1(30)}-\alpha_{1} \tilde{p}_{h_{2}-1(30)}\right) .
\end{aligned}
$$

To determine $p$, we use the condition that in the steady state the average number of busy channels is equal to the system load factor 


$$
\mathbf{E}\left(T_{\mathrm{sv}}\right)\left(\lambda \sum_{k=0}^{h_{2}-1} p_{k}+\tilde{\lambda}\left(1-\sum_{k=0}^{h_{2}-1} p_{k}\right)\right)=p_{1}+2 p_{2}+3\left(\sum_{k=3}^{h_{2}-1} p_{k}+\sum_{k=h_{1}+1}^{\infty} q_{k}\right) .
$$

The steady-state probabilities exist under the condition that $\tilde{\lambda} \mathbf{E}\left(T_{\mathrm{sv}}\right)<3$. Using the recurrence relations (25)-(27) and the normalization condition (24), we obtain the approximate values of the steady-state probabilities from the equalities:

$$
\begin{aligned}
& \frac{1}{p_{0(N)}}=1+\tilde{p}_{1(10)(N)}+\tilde{p}_{1(01)(N)}+\tilde{p}_{2(20)(N)}+\tilde{p}_{2(11)(N)}+\tilde{p}_{2(02)(N)}+ \\
& +\sum_{k=3}^{h_{2}-1}\left(\tilde{p}_{k(30)(N)}+\tilde{p}_{k(21)(N)}+\tilde{p}_{k(12)(N)}+\tilde{p}_{k(03)(N)}\right)+\sum_{k=h_{1}+1}^{N}\left(\tilde{q}_{k(30)(N)}+\tilde{q}_{k(21)(N)}+\tilde{q}_{k(12)(N)}+\tilde{q}_{k(03)(N)}\right) \\
& p_{1(10)(N)}=p_{0(N)} \tilde{p}_{1(10)(N)}, \quad p_{1(01)(N)}=p_{0(N)} \tilde{p}_{1(01)(N)}, p_{2(20)(N)}=p_{0(N)} \tilde{p}_{2(20)(N)}, \\
& p_{2(11)(N)}=p_{0(N)} \tilde{p}_{2(11)(N)}, \quad p_{2(02)(N)}=p_{0(N)} \tilde{p}_{2(02)(N)} ; \\
& p_{k(30)(N)}=p_{0(N)} \tilde{p}_{k(30)(N)}, \quad p_{k(21)(N)}=p_{0(N)} \tilde{p}_{k(21)(N)}, \quad p_{k(12)(N)}=p_{0(N)} \tilde{p}_{k(12)(N)} \\
& p_{k(03)(N)}=p_{0(N)} \tilde{p}_{k(03)(N)}, \quad 3 \leq k \leq h_{2}-1 ; \\
& q_{k(30)(N)}=p_{0(N)} \tilde{q}_{k(30)(N)}, \quad q_{k(21)(N)}=p_{0(N)} \tilde{q}_{k(21)(N)}, \quad q_{k(12)(N)}=p_{0(N)} \tilde{q}_{k(12)(N)} \\
& q_{k(03)(N)}=p_{0(N)} \tilde{q}_{k(03)(N)}, \quad h_{1}+1 \leq k \leq N ; \\
& p_{1(N)}=p_{1(10)(N)}+p_{1(01)(N)}, p_{2(N)}=p_{2(20)(N)}+p_{2(11)(N)}+p_{2(02)(N)} ; \\
& p_{k(N)}=p_{k(30)(N)}+p_{k(21)(N)}+p_{k(12)(N)}+p_{k(03)(N)}, \quad 3 \leq k \leq h_{1} ; \\
& p_{k(N)}=p_{k(30)(N)}+p_{k(21)(N)}+p_{k(12)(N)}+p_{k(03)(N)}+q_{k(30)(N)}+q_{k(21)(N)}+q_{k(12)(N)}+ \\
& +q_{k(03)(N))}, \quad h_{1}+1 \leq k \leq h_{2}-1 ; \\
& p_{k(N)}=q_{k(30)(N)}+q_{k(21)(N)}+q_{k(12)(N)}+q_{k(03)(N)}, \quad h_{2} \leq k \leq N .
\end{aligned}
$$

Using the formulas (15) we find the approximate values of the stationary characteristics $\mathbf{E}(Q), \mathbf{E}(W)$ and $\mathbf{P}_{\text {sv }}$.

\section{Examples for the calculating of stationary characteristics}

Introduce the notation for the studied queueing systems. Let the $\mathrm{M} / \mathrm{E}_{2} / 3 / \infty$ system be the System 1, the $\mathrm{M} / \mathrm{E}_{2} / 3 / \infty$ system with the threshold strategy of the random dropping of customers be the System 2 and the M/E $/ 3 / \infty$ system with the hysteretic strategy of the random dropping of customers be the System 3.

For all the systems we put: $\mu_{1}=1, \mu_{2}=2$. Let $\lambda=1.8$ for the System 1, $\lambda=2.25, \beta=0.8$ for the Systems 2 and $3, h=13$ for the System $2, h_{1}=8, h_{2}=13$ for the System 3 . 
The values of the steady-state probabilities and stationary characteristics of the systems 1-3, found using the recurrence relations obtained in this paper, are presented in Tables 1 and 2 . In order to verify the obtained values, the tables contain the computing results evaluated by the GPSS World simulation system [7] for the time value $t=10^{7}$.

In calculating the approximate values of the steady-state probabilities $p_{k}$, the number $N$ is chosen so large that the condition

$$
\mathbf{E}(Q)_{(N)}-\mathbf{E}(Q)_{(N-1)}<10^{-9}
$$

holds. The obtained minimum values of $N$, for which the condition (28) is satisfied, are equal to 156, 164 and 162 for the Systems 1, 2 and 3, respectively.

Table 1

Stationary distribution of the number of customers in the system

(1 - analytical method, 2 - GPSS World)

\begin{tabular}{|c|c|c|c|c|c|c|}
\hline \multirow{2}{*}{$k$} & \multicolumn{5}{|c|}{ Values of the steady-state probabilities $p_{k}$} \\
\cline { 2 - 7 } & \multicolumn{2}{|c|}{ System 1} & \multicolumn{2}{c|}{ System 2} & \multicolumn{2}{c|}{ System 3} \\
\cline { 2 - 8 } & $1, N=156$ & 2 & $1, N=164$ & 2 & $1, N=162$ & 2 \\
\hline 0 & 0.0241532 & 0.0241463 & 0.0019693 & 0.0019621 & 0.0028508 & 0.0028780 \\
1 & 0.0666245 & 0.0664944 & 0.0068771 & 0.0068758 & 0.0099553 & 0.0100608 \\
2 & 0.0942915 & 0.0942690 & 0.0124350 & 0.0124441 & 0.0180009 & 0.0180814 \\
3 & 0.0954951 & 0.0954166 & 0.0163353 & 0.0163913 & 0.0236470 & 0.0237228 \\
4 & 0.0881153 & 0.0881351 & 0.0197881 & 0.0198060 & 0.0286452 & 0.0287503 \\
5 & 0.0786458 & 0.0785520 & 0.0233579 & 0.0233281 & 0.0338128 & 0.0338232 \\
6 & 0.0693057 & 0.0694374 & 0.0273314 & 0.0272895 & 0.0395640 & 0.0395121 \\
7 & 0.0607719 & 0.0609322 & 0.0318850 & 0.0317958 & 0.0461397 & 0.0460776 \\
8 & 0.0531851 & 0.0530799 & 0.0371591 & 0.0368896 & 0.0536338 & 0.0536840 \\
9 & 0.0465099 & 0.0465122 & 0.0432901 & 0.0432911 & 0.0618105 & 0.0619795 \\
10 & 0.0406604 & 0.0405606 & 0.0504217 & 0.0503929 & 0.0667520 & 0.0670598 \\
20 & 0.0105844 & 0.0105622 & 0.0296765 & 0.0297640 & 0.0236203 & 0.0235319 \\
30 & 0.0027549 & 0.0027422 & 0.0077243 & 0.0078382 & 0.0061479 & 0.0060459 \\
40 & 0.0007170 & 0.0007173 & 0.0020105 & 0.0019613 & 0.0016002 & 0.0016040 \\
50 & 0.0001866 & 0.0002080 & 0.0005233 & 0.0005068 & 0.0004165 & 0.0004464 \\
60 & 0.0000486 & 0.0000583 & 0.0001362 & 0.0001378 & 0.0001084 & 0.0001119 \\
70 & 0.0000126 & 0.0000162 & 0.0000355 & 0.0000264 & 0.0000282 & 0.0000277 \\
80 & 0.0000033 & 0.0000062 & 0.0000092 & 0.0000095 & 0.0000073 & 0.0000074 \\
90 & $8.566 \cdot 10^{-7}$ & 0.0000008 & 0.0000024 & 0.0000014 & 0.0000019 & 0.0000011 \\
100 & $2.229 \cdot 10^{-7}$ & 0.0000008 & $6.251 \cdot 10^{-7}$ & 0.0000013 & $4.975 \cdot 10^{-7}$ & 0.0000002 \\
110 & $5.803 \cdot 10^{-8}$ & 0.0000000 & $1.626 \cdot 10^{-7}$ & 0.0000009 & $1.295 \cdot 10^{-7}$ & 0.0000000 \\
130 & $3.931 \cdot 10^{-9}$ & & $1.102 \cdot 10^{-8}$ & 0.0000000 & $8.773 \cdot 10^{-9}$ & \\
150 & $2.663 \cdot 10^{-10}$ & & $7.467 \cdot 10^{-10}$ & & $5.943 \cdot 10^{-10}$ & \\
\hline
\end{tabular}


Stationary characteristics of the system

\begin{tabular}{|c|c|c|c|c|}
\hline $\begin{array}{c}\text { The system } \\
\text { number }\end{array}$ & Method & $\mathbf{E}(Q)$ & $\mathbf{E}(W)$ & $\mathbf{P}_{\mathrm{sv}}$ \\
\hline 1 & analytical & 5.7429114 & 3.1905063 & 1 \\
\hline 1 & GPSS World & 5.745 & 3.193 & 1 \\
\hline 2 & analytical & 12.3769008 & 6.2553772 & 0.8793786 \\
\hline 2 & GPSS World & 12.378 & 6.256 & 0.879 \\
\hline 3 & analytical & 10.7951977 & 5.4825119 & 0.8751218 \\
\hline 3 & GPSS World & 10.790 & 5.478 & 0.875 \\
\hline
\end{tabular}

\section{Conclusions}

The numerical algorithm for solving a system of linear algebraic equations for the steady-state probabilities, proposed in this paper, is constructed taking into account the structural features of the system, in particular the presence of three or four unknown in most of its equations. The obtained recurrence relations are used for the direct calculation of the solutions of the system, that allow us to reduce the number of calculations in comparison with application of one of the classical methods (direct or iterative). Direct methods require the implementation of preliminary transformations of the matrix of the system, and only in the second stage of computing the solutions is consistently defined. When using iterative methods, the calculations are performed repeatedly until a solution is found with a given accuracy. The use of iterative methods is possible only under the condition of diagonal dominance of the matrix of the system.

\section{References}

[1] Bocharov P.P., Pechinkin A.V., Queueing Theory, RUDN, Moskow 1995 (in Russian).

[2] Brockmeyer E., Halstrøm H.L., Jensen A., The Life and Works of A.K. Erlang, Danish Academy of Technical Sciences, Copenhagen 1948.

[3] Chydziński A., Nowe modele kolejkowe dla węzłów sieci pakietowych, Pracownia Komputerowa Jacka Skalmierskiego, Gliwice 2013 (in Polish).

[4] Tikhonenko O., Kempa W.M., Queue-size distribution in M/G/1-type system with bounded capacity and packet dropping, Communications in Computer and Information Science 2013, 356, 177-186.

[5] Zhernovyi Yu., Kopytko B., Zhernovyi K. On characteristics of the M $1 / \mathrm{G} / 1 / \mathrm{m}$ and $\mathrm{M} \theta / \mathrm{G} / 1$ queues with queue-size based packet dropping, Journal of Applied Mathematics and Computational Mechanics 2014, 13(4), 163-175.

[6] Zhernovyi Yu.V., Zhernovyi K.Yu., Potentials method for $\mathrm{M} / \mathrm{G} / 1 / \mathrm{m}$ systems with threshold operating strategies, Cybernetics and Systems Analysis 2016, 52, 3, 481-491.

[7] Zhernovyi Yu., Creating Models of Queueing Systems Using GPSS World, LAP Lambert Academic Publishing, Saarbrücken 2015. 